\title{
A method for detecting IL-6 in serum of patients with uremia
}

\author{
Qingbo $\mathrm{Bi}^{1,}{ }^{*}$, and Shih-Mo Yang ${ }^{2}$ \\ ${ }^{1}$ Shanghai University, Shanghai, China \\ ${ }^{2}$ Shanghai University, Shanghai, China
}

\begin{abstract}
As the gold standard of protein detection, enzyme-linked immunosorbent assay (ELISA) is widely used in medical treatment and biology. Here, we report a digital ELISA method that combines fluorescence-coded magnetic beads with micropore arrays to effectively improve the accuracy of the detection. Fluorescence coded magnetic beads were used as solid support of ELISA, which were modified to specifically capture IL-6 in serum, and then combined with galactosidase to form a sandwich structure. These beads are then mixed with a fluorescent substrate and passed into a microfluidic chip. Under the action of gravity, the beads are trapped and isolated by an array of micropores in the chip. Combined with image recognition technology, the fluorescence intensity of micropores containing enzymes will increase rapidly. By mining image information, the IL-6 content in uremia patients can be detected with high precision.
\end{abstract}

\section{Introduction}

Protein is an important component of human body and participates in many life activities of human body. Many proteins exist in the human body in extremely small amounts. The improvement of protein detection technology is of great significance to disease detection and drug discovery[1]. Current common protein detection methods, such as fluorescence spectrometry[2], spectrophotometry[3], enzyme-linked immunosorbent assay[4]. Conventional ELISA tests spread the fluorescence product into a large container, and the instrument cannot read the data effectively when the protein concentration is too low. At present, there are many ways to amplify the fluorescence signal generated during protein detection[5-7]. Digital ELISA uses the method of oil seal to divide the immune complex into the micropores of femtoliter[8, 9], which can detect the output of fluorescent substances in tens of thousands of micropores in a single detection, greatly improving the detection accuracy.The digital ELISA used in this paper achieved the detection level of $\mathrm{pg} / \mathrm{mL}$ by constructing the relationship between the proportion of active micropores and protein concentration.

As an important cytokine in the human body, IL-6 is closely related to kidney disease[10] and plays a key role in the maturation of B cells, as one of the cytokines driving acute inflammatory response[11].The continuous development of chronic kidney disease to end-stage outcome is uremia, clinical manifestations of renal function damage, metabolic disorder, seriously affect the patient's physical and mental health. Decline in renal function will lead to lymphocytosis and abnormal levels of interleukin[12-15]. Serum IL-6 concentrations in 10 healthy people and 20 uremia patients were determined by a new method and the results were compared.

\section{Materials and methods}

\subsection{Acquisition of reagents and serums}

In this experiment, fluorescent coded magnetic beads that can specifically capture IL- 6 and the required PBS buffer, IL-6 standard solution, detection antibody solution and other reagents were all purchased from ColorTech Company. Serum of patients and normal subjects were provided by Wuxi People's Hospital. The test reagents were stored in a refrigerator at $4{ }^{\circ} \mathrm{C}$, and the serum samples were frozen at $-20^{\circ} \mathrm{C}$.

\subsection{System structures}

We designed a detection system (Figure 1), which mainly includes four parts: liquid control device, microfluidic chip, fluorescence imaging device, and upper machine control system. This platform can help us quickly get fluorescence images and analyze the images. The liquid sampling device is controlled by STM32 microcontroller and connected with the upper machine through serial communication. Fluorescence imaging device is composed of black and white CCD camera, micro lens, laser transmitter. Image analysis program is run on the upper machine.

\footnotetext{
* Corresponding author: 19721848@shu.edu.cn
} 


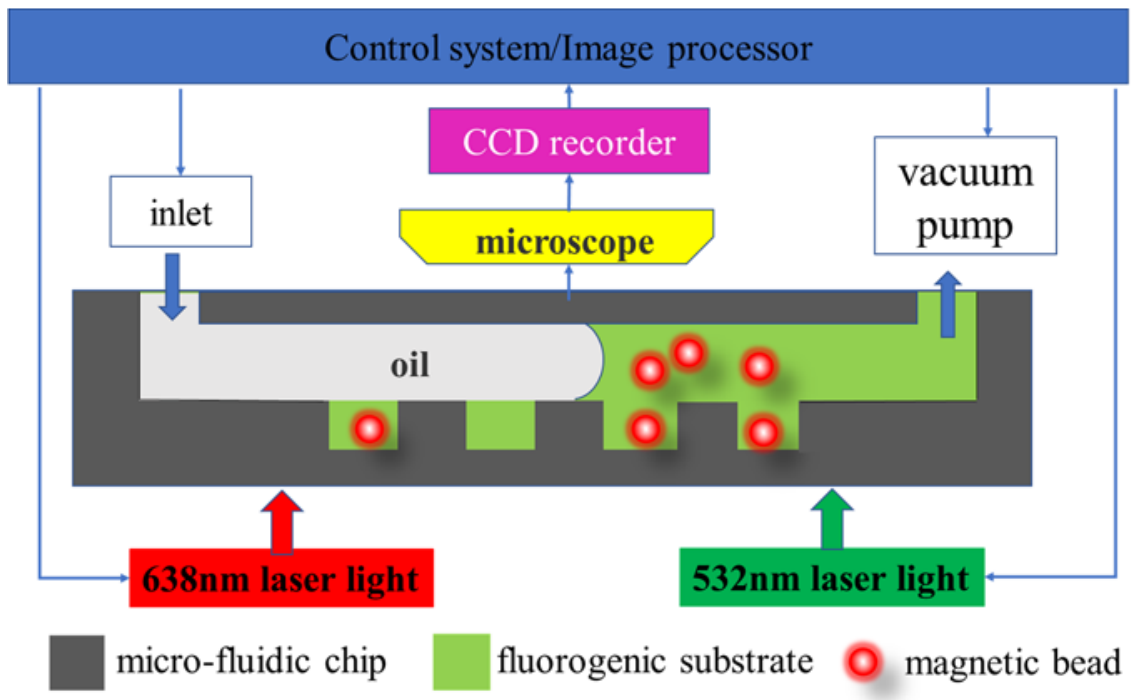

Fig. 1. Schematic diagram of detection system.

\subsection{Magnetic beads to capture}

The IL-6 standard was diluted to obtain six known sample solutions of different concentrations. The serum was removed from the refrigerator, thawed at room temperature, centrifuged at $10000 \mathrm{r} / \mathrm{min}$ for 5 minutes, and its supernatant was diluted and tested. The captured microsphere suspension was mixed with detection antibody solution and then mixed with sample solution or serum to be tested. After the solution was evenly mixed by pipettor, the solution was left standing and incubated for half an hour at room temperature under dark conditions. Use a magnetic stand to absorb and discard the supernatant, and use a buffer to clean the magnetic beads. Then streptavidin and $\beta$-galactosidase $(\mathrm{S} \beta \mathrm{G})$ solution was added, pipetted evenly with pipettor and incubated for 5 minutes. After cleaning the magnetic beads with buffer solution again, the excess liquid is discarded, and the vibration is uniform. The magnetic beads suspension is sent into the system for detection with pipettor.

\subsection{Image acquisition}

The magnetic beads to be detected are suspended in the inlet of the microfluidic chip, and the liquid sampling device applies a vacuum at the outlet of the microfluidic chip, and the magnetic beads suspension is sucked into the microflow channel. After waiting for two minutes, the magnetic beads were captured by an array of micropores in the microflow channel under the action of gravity. Since the radius of the micropores is $2 \mu \mathrm{m}$, while the radius of the magnetic beads is about $1.5 \mu \mathrm{m}$, each magnetic bead was separated in a different chamber by the micropores. Then the fluorocarbon oil is dropped at the entrance, and the fluorocarbon oil flows through the array of micropores under the action of vacuum to separate the different microreaction chambers.

The CCD camera first takes the fluorescence image of the magnetic bead itself excited by the laser at $638 \mathrm{~nm}$. Then, the first micropore fluorescence image was excited by $532 \mathrm{~nm}$ laser, and the second micropore fluorescence image was taken one minute after the enzyme catalyzed the fluorescent substrate. The fluorescence intensity of micropores containing enzymes will be significantly enhanced.

\subsection{Image processing}

The original fluorescence image may produce various noises due to equipment reasons[16], and the impurities accidentally dropped into the experimental operation and the bubbles not excluded in the flow passage may also cause the image to appear large area of light spots.

Firstly, median filtering was used to remove isolated noise points on the image, and then the area size of each spot was obtained through the edge detection algorithm. An appropriate threshold was selected to limit the area of the spot, remove the spot generated by impurities and bubbles, and select the spot with the appropriate size by using a square box, as shown in Figure 2a. Processing fluorescent images of the beads themselves can tell which pores are trapping the beads. Since the position of the magnetic bead has been fixed, we selected the captured fluorescence photos of micropores using the box with the same position, as shown in Figure $2 \mathrm{~b}$ and Figure 2c. All pixel values in the box were added up to the fluorescence intensity value of each micropore. In a sample test, we can obtain nearly ten thousand micropore fluorescence intensity values of captured magnetic beads from an image. The increase value of fluorescence intensity was obtained by subtracting the corresponding fluorescence intensity of each micropore before and after enzyme catalysis. 
Finally, K-means clustering was used for the increase value of fluorescence intensity of micropores, which were divided into active and inactive micropores. Among them, the pores with a greater increase in fluorescence intensity were the active pores. The ratio of active pores in each experiment, which is the ratio of the number of micropores containing the enzyme to the total number of micropores captured by the magnetic beads, was recorded.
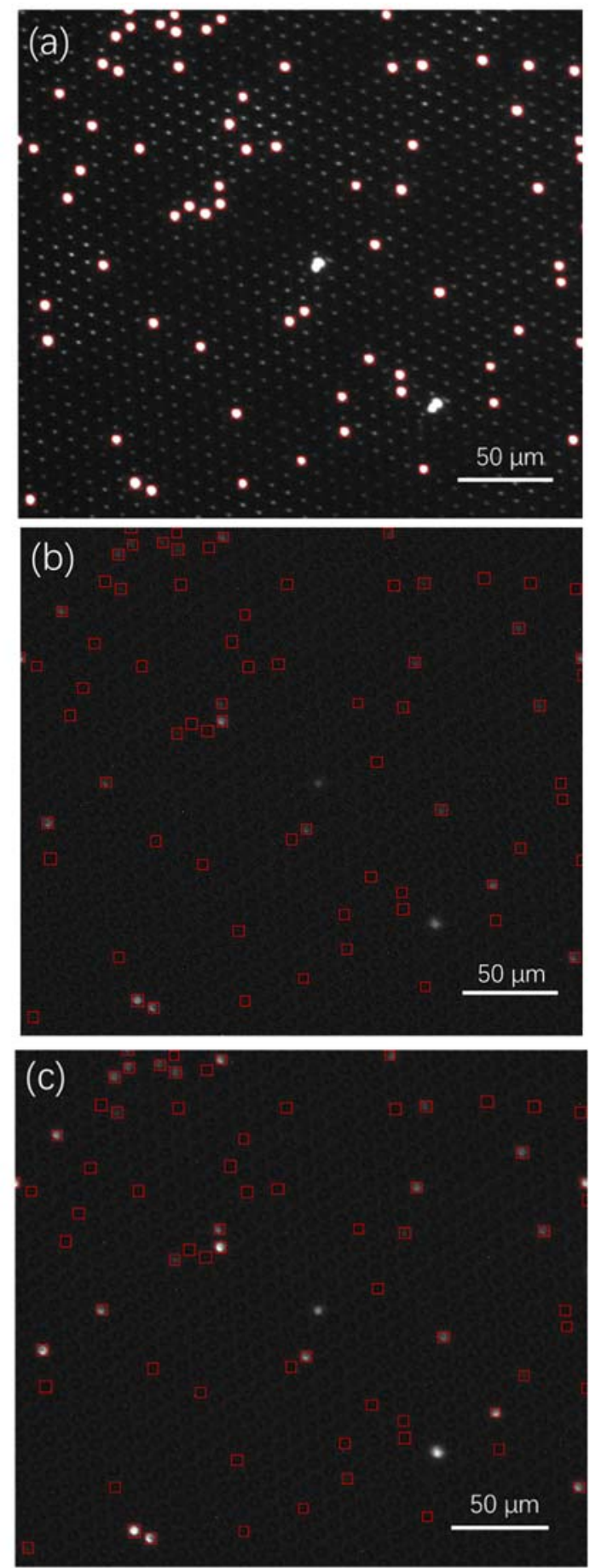

Fig. 2. Select the spot of appropriate size in the fluorescence image. (a) Fluorescence generated by the fluorescent substance added in the excitation magnetic bead; (b) Fluorescence generated by fluorescence substances in micropores; (c) After catalyzed by the enzyme for 1 minute, the fluorescence generated by the fluorescent material in the micropore was excited.

\section{Results}

\subsection{Standard curve drawing}

We used the above method to detect six groups of IL-6 at known concentrations between 0 and $1 \mathrm{pg} / \mathrm{mL}$, and recorded the corresponding active pore ratios. Then, the least square method was used to fit the standard curve between IL- 6 concentration and the proportion of active pores, as shown in Figure 3 , and the $R^{2}$ value of the fitting line was 0.996. By detecting the proportion of active pores in the serum to be tested and substituting it into the standard curve, the concentration value of IL-6 in the tested sample can be obtained.

The limit of detection (LOD) is the quantity when the response signal generated by the detector is three times the noise value, which is one of the important indicators of sensitivity[17]. In this experiment, we repeatedly tested the standard deviation of the proportion of active Wells when IL-6 concentration was $0 \mathrm{pg} / \mathrm{mL}$, and obtained the value of LOD of $0.12 \mathrm{pg} / \mathrm{mL}$, which proved that this method could realize the accurate test of $\mathrm{pg} / \mathrm{mL}$.

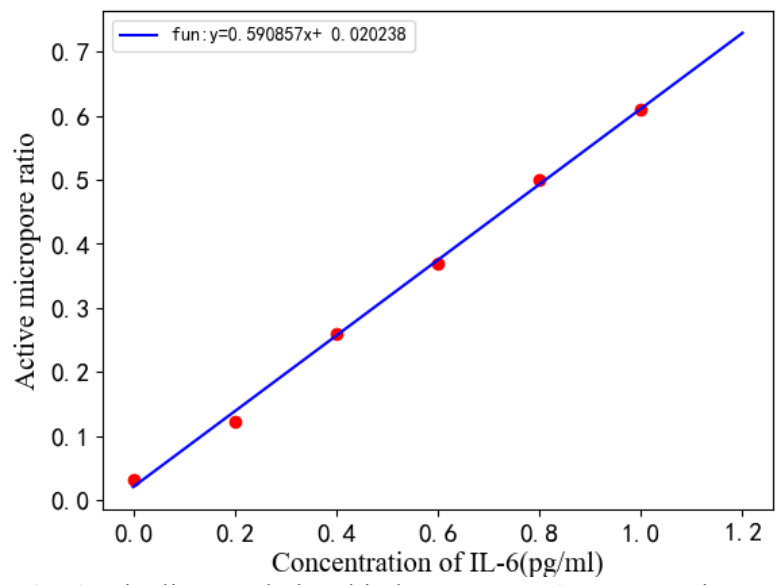

Fig. 3. The linear relationship between IL-6 concentration and the proportion of active micropores in the micropores captured by magnetic beads.

\subsection{Serum detection}

There were no malignant tumor patients, autoimmune disease patients, AIDS patients in the patient group and healthy group, and no recent use of drugs affecting the autoimmune system. The healthy group had normal renal function, while the patient group had end-stage renal disease. The average glomerular filtration rate, urea nitrogen level and creatinine level in the patient group were $4.72 \pm 2.41 \mathrm{~mL} / \mathrm{min}, 24.18 \pm 7.44 \mathrm{mmol} / \mathrm{L}$ and 984.6 $\pm 306.21 \mu \mathrm{mol} / \mathrm{L}$, respectively.

We detected the sera of 10 healthy people and 20 uremia patients. The data obtained were analyzed using SPSS19.0 software, and the results were expressed as mean \pm standard deviation. The resulting statistics are shown in Table 1. There were 9 males and 11 females with an average age of 53.35 years in the uremia patients, and 5 males and 5 females in the healthy control group with an average age of 50.1 years. There was no 
significant difference in age between the uremia patients and healthy controls $(\mathrm{P}>0.05)$. The results showed that there was no significant difference in serum IL-6 levels between the two sexes $(\mathrm{P}>0.05)$, and the serum IL-6 concentration of uremia patients was significantly higher than that of healthy controls $(\mathrm{P}<0.01)$.

Table 1. Serum test information results.

\begin{tabular}{|c|c|c|}
\hline Lab test & $\begin{array}{c}\text { health controls } \\
(\mathrm{n}=10)\end{array}$ & $\begin{array}{c}\text { Uremia patients } \\
(\mathrm{n}=20)\end{array}$ \\
\hline Age(years) & $50.1 \pm 7.4$ & $53.35 \pm 13.59$ \\
\hline $\begin{array}{c}\text { IL-6 levels in } \\
\text { male (pg/mL) }\end{array}$ & $3.97 \pm 2.92$ & $17.7 \pm 25.96$ \\
\hline $\begin{array}{c}\text { IL-6 levels in } \\
\text { female (pg/mL) }\end{array}$ & $3.22 \pm 2.22$ & $16.9 \pm 18.62$ \\
\hline $\begin{array}{c}\text { IL-6 levels } \\
(\mathrm{pg} / \mathrm{mL})\end{array}$ & $3.59 \pm 2.48$ & $17.3 \pm 21.59$ \\
\hline
\end{tabular}

\section{Conclusion}

We used fluorescence-coded magnetic beads as solid support to specifically capture IL-6. Approximately 80,000 micropores with a diameter of about $4 \mu \mathrm{m}$ were used to capture magnetic beads, and the fluorescent substrates in each micropore were separated into separate reaction chambers using fluorocarbon oil. When the concentration of the protein to be detected is low, each magnetic bead binds one or zero catalytic enzymes. The fluorescence intensity of the captured catalytic enzyme micropore will increase rapidly. Based on OpenCV, we analyzed the fluorescence intensity of each micropore captured by micromagnetic beads, which was divided into active micropores and inactive micropores by clustering algorithm. The function relationship between the proportion of active micropores and protein concentration was established, and the high precision detection of IL- 6 was finally realized. In addition, our system clinically detected the IL- 6 concentration in 20 uremia patients and 10 healthy controls, and it was confirmed that the IL-6 concentration in uremia patients was high, and there was no significant relationship between the IL- 6 concentration and gender.

Although the digital ELISA experiment we implemented in this study can accurately detect IL-6, the detection range still needs to be improved. With the increase of sample concentration, the proportion of active micropores could not exceed $100 \%$. When the sample concentration is greater than $1.2 \mathrm{pg} / \mathrm{mL}$, the relationship between the concentration of active pores and the sample concentration will be greatly deviated from the linear equation fitted. Therefore, the serum needs to be diluted first when detecting the serum, and the dilution operation will slightly reduce the detection accuracy. In addition, fluorescent coded magnetic beads can distinguish different bead groups by adding different dyes. In future studies, we will use color CCD camera to distinguish bead groups that capture different proteins by judging the color of magnetic beads, so as to realize multiple detection of proteins.

\section{Acknowledgements}

Thanks to ColorTech (Suzhou) Company for providing some technical support and Wuxi People's Hospital for the extraction of patients' serum.

\section{References}

1. L. Cohen, D.R. Walt, Chem Rev 119, 293-321 (2019)

2. J.R. Wisniewski, F.Z. Gaugaz, Anal Chem 87, 4110-4116 (2015)

3. Q.S. Wang, Q.Z. Zhai, J. Luo, Asian J Chem 25, 5470-5472 (2013)

4. S. Y. Toh, M. Citartan, S. C. B. Gopinath, T. H. Tang, Biosens Bioelectron 64, 392-403 (2015)

5. R. de la Rica, M.M. Stevens, Nat Nanotechnol 7, 821-824 (2012)

6. J.H. Liang et al., J Immunol Res 2019, 5474519 (2019)

7. K. Iha, M. Inada, et al., Expert Rev Mol Diagn 13, 10-10 (2013)

8. C. W. Kan et al., Lab Chip 12, 977-985 (2012)

9. H. B. Zhang, S. Nie, C. M. Etson, R. M. Wang, D. R. Walt, Lab Chip 12, 2229-2239 (2012)

10. C. Martos-Rus et al., Sci Rep-Uk 11, 2974 (2021)

11. W.E. Naugler, M. Karin, Trends Mol Med 14, 109-119 (2008)

12. M.G.H. Betjes, Toxins 12, 224 (2020)

13. Y. Romanova et al., Biomolecules 10, 257 (2020)

14. K.A. Lisowska et al., Sci Rep-Uk 9, 5041 (2019)

15. M. Diaz-Ricart et al., Toxins 12, 361 (2020)

16. E.R. Fossum, D.B. Hondongwa, Ieee J Electron Devi 2, 33-43 (2014)

17. C.I. Tobos et al. J Immunol Methods 474, 112643 (2019) 